\title{
Dandy-Walker Malformation in an Asymptomatic 27-Year-Old Woman. A Case Report
}

Joyce Antonella Jiménez,' Daniel Francisco Landívar,' Fernando Xavier Posligua, ' Jorge Rigoberto González.'

\begin{abstract}
Introduction: Dandy-Walker malformation (DWM) is a rare congenital disorder that involves the cerebellum and fourth ventricle. Incidentally detected asymptomatic DWM is sparsely reported in literature at extremes of age (from 1 to 75 years) in association with different diseases. We aim to report the case of a patient with an incidental finding of a DWM after a road traffic accident, reinforcing the importance of suspicion, investigation, diagnosis of clinical presentations. The Case: This case report describes a 27-year-old female patient who presented to the emergency room after a motor vehicle collision. She suffered a left kidney and spleen injury. The patient's brain CT scan revealed an enlarged cisterna magna with normal cerebellum, partial hypoplasia of the cerebellar vermis and enlargement of the fourth ventricle compatible with DWM. Neurological examination was unremarkable. An exploratory laparotomy was performed, and the left kidney and spleen showed hemorrhage, so a left nephrectomy and splenectomy was done. Conclusions: This case report aimed to characterize the DWM, which is a congenital malformation of the central nervous system. Asymptomatic patients do not require surgical treatment; however, these patients should be followed up once a year.
\end{abstract}

Keywords: Dandy-Walker Syndrome; Dandy-Walker Variant; Hydrocephalus; Cerebellar Vermis Hypoplasia (Source: MeSH-NLM).

\section{Introduction}

Dandy-Walker Malformation (DWM) is a congenital malformation that occurs during embryonic development of the cerebellum and 4th ventricle, and has a prevalence of 1 out of 25000-30000 live births. ${ }^{1}$ The classic anatomic hallmarks of DWM are hypoplasia of the cerebellar vermis, anterior-posterior enlargement of the posterior fossa, upward displacement of the torcula and transverse sinuses, and cystic dilatation of the fourth ventricle. ${ }^{2-3}$ In addition to the classic findings that define it, DWM is related to many other abnormalities and malformations in the central nervous system (CNS) including agenesis of corpus callosum, heterotopias, occipital meningocele, visual deficits, and epilepsy. 4

The clinical presentation of DWM can be variable. Hydrocephalus is not uncommon prenatally or during the neonatal period, although this is a complication rather than part of the disease. Most cases are diagnosed during infancy. Infants may present with early signs such as somnolence, vomiting, convulsions, irritability, lack of muscle coordination and unsteadiness. Older patients might be asymptomatic with normal or near-normal neurological examinations. They usually present with neurological manifestations such as developmental delay, spasticity, poor head control and seizures.

This report describes a 27-year-old female with incidental findings of hypoplasia of the inferior vermis and an enlargement of the cisterna magna after a motor vehicle collision. The patient never developed important symptoms and signs of cerebellar involvement.

\section{The Case}

This patient is a 27-year-old Latin American female who presented to the emergency room at the Teodoro Maldonado Carbo Hospital in Ecuador with cervical, posterior left-side thoracic and abdominal pain after a road traffic accident while her husband was driving. She was in the front passenger seat of the car when they were hit by a bus driver.
She reported no relevant past medical history except for irritability, drowsiness and a caesarean section 4 years ago. She refers no allergies. Her family medical history includes a mother with a history of anxiety and hypertension. Her mother did not provide any history of medication during pregnancy. She was born at term and was the first child of nonconsanguineous parents and the only affected case in the family. Her social history is important for occasional alcohol use. She lives with her husband and son, and is psychosocially independent.

At the time of the incident, the seat belt was not fastened and the patient reports that she lost consciousness for a few minutes.

Upon arrival to the emergency room (ER), the patient was pale and noted to be tachypneic with an oxygen saturation of $98 \%$ on room air. Clasgow Coma Scale was 15. Vital signs in the ER showed a blood pressure of $90 / 63 \mathrm{mmHg}$, heart rate of 112 , respiratory rate of 23 and temperature of $97.5^{\circ} \mathrm{F}$. Blood sampling was done, and fluid and blood resuscitation were continued.

The patient's ER workup further revealed a creatinine of $0.72 \mathrm{mg} / \mathrm{dL}$, urea of $23 \mathrm{mg} / \mathrm{dL}$, sodium of $141 \mathrm{mEq} / \mathrm{L}$, potassium of $4.10 \mathrm{mEq} / \mathrm{L}$, INR of 1.04, Prothrombin Time of $12.6 \mathrm{~s}$, Partial Thromboplastin Time of 26.7 $\mathrm{s}$, white blood cells of 14.850 cells $/ \mathrm{mm}^{3}$, platelets of 120.000 cells $/ \mathrm{mm}^{3}$, hematocrit of $32.3 \%$ and hemoglobin of $10.3 \mathrm{~g} / \mathrm{dL}$.

Additionally, a FAST (Focused assessment with sonography for trauma) was performed which revealed echo-free space around the left kidney and the spleen (370 cc of free fluid). Contrast Computerized Tomography (CT) examination revealed bloody ascites around the left kidney and the spleen and we diagnosed intra-abdominal bleeding. Figure 1. Sagittal (Top) and Axial (Bottom) CT Images Demonstrating Cystic Dilatation of IV Ventricle, a Ciant Cisterna Magna and a Hypoplastic Cerebellum.

${ }^{1}$ Universidad Católica de Santiago de Guayaquil, Guayaquil, Ecuador.

About the Author: Joyce Antonella Jiménez is a recently graduated MD at Universidad Católica de Santiago de Guayaquil. She is currently studying for the United States Medical Licensing Examination to pursue her career in the neurology field.

Correspondence:

Joyce Antonella Jiménez

Address: Universidad Católica de Santiago de Guayaquil, Guayaquil, Ecuador.
Editor: Mihnea-Alexandru Găman Submission: Jun 4, 2018 Acceptance: $\operatorname{Sep} 29,2018$ Publication: Nov 9, 2018 Process: Peer-reviewed 

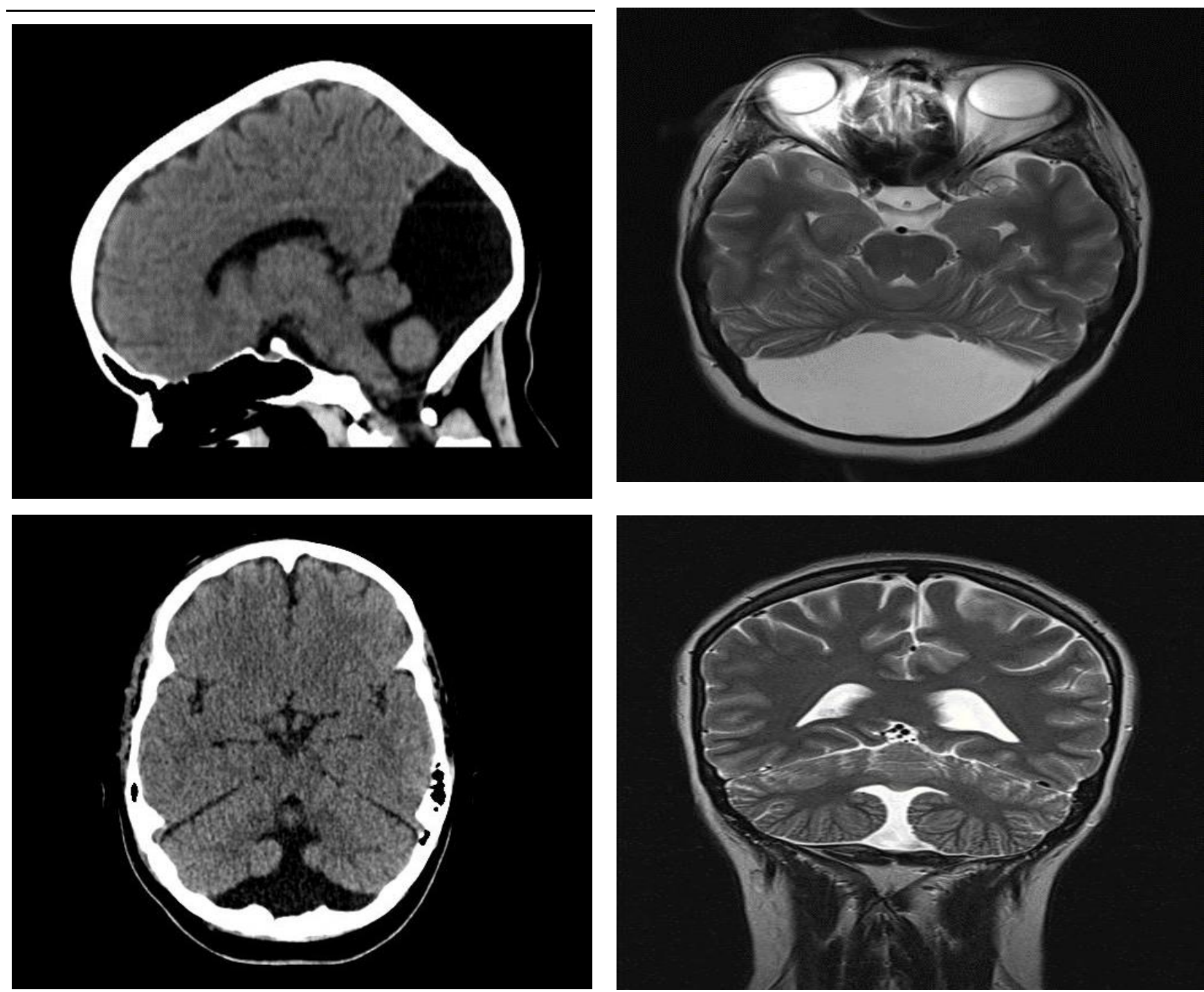

Her skull CT scan showed an enlarged cisterna magna, partial hypoplasia of the cerebellar vermis and enlargement of the fourth ventricle (Figure 1). The cerebral parenchyma appeared normal with no focal lesions or midline shifts. This suggested a mild form of Dandywalker syndrome.

Neurological examination and electroencephalogram (EEG) were unremarkable. Magnetic Resonance Imaging (MRI) to confirm these findings was suggestive for DWM (Figure 2). The patient was evaluated by the Neurosurgical team before the surgery and no intervention was recommended.

An exploratory laparotomy was performed with the left kidney and spleen showing signs of hemorrhaging. Subsequently, a left nephrectomy and splenectomy was carried out.

The patient had an uneventful post-operative recovery and went home on post-operative day 10 . Further investigations with MRI were carried without detecting any anomaly related to DWM. The patient remained without signs of disease after one year of follow-up.

Figure 2. Axial (Top) and Coronal (Bottom) Brain MRI Showing a Large Fourth Ventricle and a Small Cerebellar Vermis in T2 Weighted Image.

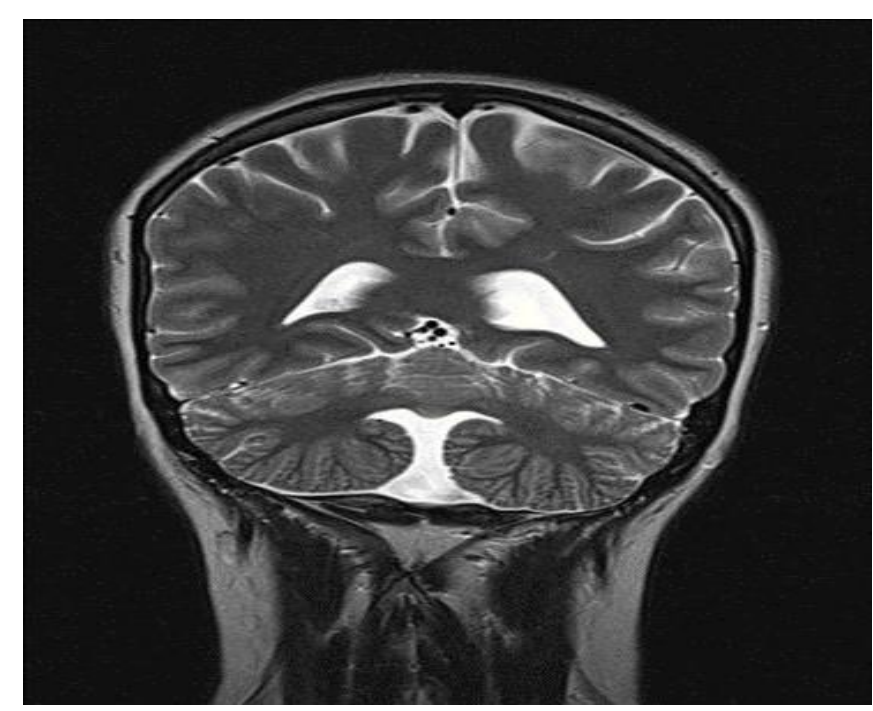

\section{Discussion}

The patient described in this case conforms to what is defined in the literature regarding the imaging characteristics for the diagnosis of DWM. The radiological images show the classic triad formed by the cystic dilation of the fourth ventricle, complete or partial agenesis of the cerebellar vermis and an enlarged posterior fossa. ${ }^{6}$ Many concomitant problems may be present, but the syndrome exists whenever these three features are found. Approximately $70 \%-90 \%$ of patients have hydrocephalus, which often develops postnatally. However, the patient in this case study did not present with this clinical feature. DWM may be associated with atresia of the foramen of Magendie and the foramen of Luschka.?

Depending on the time of onset and degree of hydrocephalus, the age at diagnosis varies from birth to older childhood. Presentation in adulthood has been reported but is infrequent. The feasible reason that may explain the mild or absence of clinical expression is the preserved cortical cytoarchitecture and the rarity of additional neurodevelopmental changes in DWS adults, compared with DWS infants. ${ }^{8}$

The clinical manifestations in symptomatic individuals include psychomotor and growth retardation, strabismus, myopia, a short neck, brachycephaly, hypertelorism, microcephaly, hypotonia, antimongoloid 
slant of palpebral fissures, large mouth with down turned corners, globulus large nose, poorly lobulated ears, high arch palate, cleft palate, small hands and feet, clinodactyly and the brachymesophalangy of the little fingers. ${ }^{9-10}$

The clinical spectrum of DWM is broad with a varying degree of neurological impairment. Dandy-Walker Variant (DWV) is a less severe form of the spectrum of DWM. Patients with DWV are more likely to present in adulthood than in infancy or childhood. The isolated DWV abnormality has the highest incidence of survival and there are reported cases of people who have had DWV their entire lives without any symptoms, such as the patient in this case study. Individuals with DWV are more likely to present in adulthood than in infancy or childhood, as presented in this case. ${ }^{11-12}$

The diagnosis of DWM can be made by ultrasonography as early as 14 weeks gestation. ${ }^{13}$ Common ultrasonographic findings include the presence of a large posterior fossa cyst, absent cerebellar vermis and splayed cerebellar hemispheres. ${ }^{14}$ Diagnostic tools after the infant is born differed from prenatal diagnostic tools for detecting DWM. Those tools included CT scans and MRI. In infants and adults, investigations for the diagnosis of these malformations are cranial CT and brain MRI. Brain MRI is the optimal exploration for the differentiation of DWM from other posterior fossa pathologies. Usually, the MRI investigation is required for better anatomic resolution prior to surgical intervention. ${ }^{15}$

For symptomatic patients, the most effective treatment incorporates the partial resection of the arachnoid membranes that constitute the "lining" of the cyst accompanied by the simultaneous insertion of a "shunt" to divert the CSF to the peritoneal cavity from where the fluid can be harmlessly reabsorbed. In this patient who was an asymptomatic DWM case, there is no extensive literature to review for their treatment. In our judgment, follow-up is the most important measure. Asymptomatic DWM patients who have been confirmed should be followed up once a year. ${ }^{16}$

The content of the follow-up should include, at least, the following three aspects: CT of the head to monitor the degree of hydrocephalus and to assess if hydrocephalus has increased; inspection of symptoms suggestive of increased intracranial pressure includes severe headaches, vomiting, and papilledema; and examination of cerebellum symptoms is also noteworthy. Cerebral trauma and intracranial infection may change the path of cerebrospinal fluid circulation in asymptomatic DWM patients. ${ }^{17}$

\section{Conclusion}

Although the DWM is rarely overlooked in early childhood, there are individuals who meet the criteria for diagnosis without clinical presentation throughout their lives. These cases should not receive pharmacological or surgical treatment, however, should be followed up due to the possibility of the onset of symptoms in later stages of life.

\section{Consent}

Written informed consent was obtained from the patient for publication of this case report and any accompanying images. A copy of the written consent is available for review by the Editor-in-Chief of this journal. 


\section{Case Report}

\section{References}

1. Forzano F, Mansour S, lerullo A, Homfray T, Thilaganathan B. Posterior fossa malformation in fetuses: a report of 56 further cases and a review of the literature. Prenat Diagn. 2007 Jun;27(6):495-501.

2. Klein O, Pierre-Kahn A, Boddaert N, Parisot D, Brunelle F. Dandy-Walker malformation: prenatal diagnosis and prognosis. Childs Nerv Syst. 2003 Aug;19(78):484-489.

3. Correa CG, Amaral LF, Vedolin LM. Neuroimaging of Dandy-Walker malformation: new concepts. Top Magn Reson Imaging. 2011 Dec;22(6):303-312.

4. Maria BL, Zinreich SJ, Carson BC, Rosenbaum AE, Freeman JM. Dandy-Walker syndrome revisited. Pediatr Neurosci. 1987 Feb;13(1):45-51.

5. Al-Turkistani HK. Dandy-Walker syndrome. J Taibah Univ Sci. 2014 Jul;9(3):209-212.

6. Chane VR, Patra KC, Meshram N, Chauhan A, Kalbhande A, Wankhede S. Dandy Walker variant mimicking as cerebral palsy with severe neurological impairment. Int J Res Med Sci. 2014 Aug;2(3):1191-1193.

7. Andrade N, Karande V. Dandy-Walker Syndrome with Giant Cell Lesions and Cherubism. Ann Maxillofac Surg. 2018 Jan-Jun;8(1):131.

8. Belfquih H, Elmostarchid B. Asymptomatic Dandy-Walker syndrome in an adult. Pan Afr Med J. 2014 Sep;19.

9. Tadakamadla J, Kumar S, Mamatha CP. Dandy-Walker malformation: An incidental finding. Indian J Hum Cenet. 2010 Jan-Apr;16(1):33-35.
10. Alexiou CA, Sfakianos G, Prodromou N. Dandy-Walker malformation: analysis of 19 cases. J Child Neurol. 2010 Feb;25(2):188-191.

11. Dawra RD, Karia S, Shah N, Desousa A. Psychosis in a Case of Dandy-Walker Syndrome: A Case Report. J Clin Diagn Res. 2017 May;11(5): VDo3-VDo4.

12. Álvarez E, Schadegg D, Bengaly M, Carcía-Arilla E. [Dandy-Walker syndrome in an independent for basic activities of daily living woman of 85 years old] Rev Esp Ceriatr Cerontol. 2018 Mar-Apr;53(2):115-117. Spanish

13. Leibovitz Z, Haratz KK, Malinger G, Shapiro I, Pressman C. Fetal posterior fossa dimensions: normal and anomalous development assessed in mid-sagittal cranial plane by three-dimensional multiplanar sonography. Ultrasound Obstet Gynecol. 2014 Feb;43(2):147-153.

14. Candolfi Colleoni G, Contro E, Carletti A, Ghi T, Campobasso G, Rembouskos G, Volpe $G$, Pilu G, Volpe P. Prenatal diagnosis and outcome of fetal posterior fossa fluid collections. Ultrasound Obstet Cynecol. 2012 Jun;39(6):625-631.

15. Jurcă MC, Kozma K, Petcheşi CD, Bembea M, Pop OL, MuTiu C, Coroi MC, Jurcă AD, Dobjanschi L. Anatomic variants in Dandy-Walker complex. Rom J Morphol Embryol. 2017 Sep;58(3):1051-1055.

16. Aminoff M, Daroff R. Encyclopedia of The Neurological Sciences. 2nd ed. Burlington: Elsevier Science; 2014 May; p.935-941.

17. Li J, Hu Q, Yan F, Shrestha S, Chen G. An Asymptomatic Dandy-Walker Malformation-A Case Report and Literature Review. Neurosurg Q. 2016 Feb;26(1):87-89.

\section{Acknowledgments}

None.

Conflict of Interest Statement it Funding

The Authors have no funding, financial relationships or conflicts of interest to disclose.

Author Contributions

Conceptualization: JAJ. Investigation: JAJ. Resources: FXP. Writing - Original Draft: JAJ, DFL, FXP, and JRG. Writing - Review A Editing: JAJ, DFL, and FXP. Visualization: JAJ. Supervision: JAJ.

Cite as:

Jiménez J, Landívar D, Posligua F, González J. Dandy-Walker Malformation in an Asymptomatic 27-Year-Old Woman. A Case Report. Int J Med Students. 2018 Sep-Dec;6(3):110-113.

This work is licensed under a Creative Commons Attribution 4.0 International License 